\title{
Feature extraction of gear fault signal based on Sobel operator and WHT
}

\author{
Jian-Hua Cai* and Wei-Wen Hu \\ Department of Physics and Electronics, Hunan University of Arts and Science, Changde, Hunan, China
}

Received 13 July 2012

Revised 21 November 2012

Accepted 27 November 2012

\begin{abstract}
Taking Wigner-Ville distribution of gear fault signal as a picture, Sobel operator was applied for edge detection of picture and then Hough transform was used to extract signal feature. Some simulated and measured signals have been processed to demonstrate the effectiveness of new method, which was compared with traditional Wigner-Hough transform and SPWDHough transform. The results show that the proposed method can suppress cross term which is produced from using Wigner-Ville distribution to analyze multi-component signal, especially under the condition of low signal to noise ratio. The improved WignerHough transform can effectively suppress the influence of noise and has a good real-time performance because its algorithm is fast. The proposed method provides an effective method to determine the state of gear accurately.
\end{abstract}

Keywords: Wigner-Ville distribution, hough transform, Sobel operator, feature extraction of gears

\section{Introduction}

Because time-frequency analysis can locate information both in time domain and frequency domain, it has become a main tool for processing non-stationary signal and is commonly used in equipment fault diagnosis, such as wavelet analysis, WVD (Wigner-Ville distribution) and recently-developed Hilbert-Huang transform. These have been applied widely in equipment fault diagnosis field [1-4]. In literature [5], time-frequency spectrum was taken as 2-d image and Hough transform was applied for further fault diagnosis. Wigner-Hough transform was researched by using state recognition of rolling bearing to confirm its effect. But in lower SNR (signal to noise ratio), WignerHough transform will be submerged by noise and it is hard to finish the extraction of signal feature. The author proposed an improved WHT (Wigner-Hough transform) based on Sobel filter operator. Before using Hough transform, edge detection of time-frequency distribution firstly was applied for fault signal to reduce noise influence, followed by use of Hough transform and to extract the feature of fault signal rapidly. Results show that method proposed in this paper can not only accurately extract fault signal features even in low SNR but its operation speed is faster than that of WVD in the same conditions.

*Corresponding author: Jian-Hua Cai, Department of Physics and Electronics, Hunan University of Arts and Science, Changde 415000, Hunan, China. Tel.: +86073 6718 6121; Fax: +86 0736718 6121; E-mail: cjh1021cjh@163.com. 


\section{Wigner-Hough transform}

\subsection{Hough transform}

Hough transform was mainly used to detect straight line. Its principle was shown in Fig. 1 [6,7]. In plane $(x, y)$, straight line L can be represented as in the following equation.

$$
\rho=x \cos \theta+y \sin \theta, \quad 0 \leqslant \theta \leqslant \pi
$$

Equation (1) is a polar coordinate equation for line L. $\rho$ represents the distance from line to origin. $\theta$ is the angle between $x$ axis and the vertical. Through the mapping of Eq. (1), each point in panel $(x, y)$ corresponds to a sine curve in parameter space $(\rho, \theta)$ where the curve corresponding to each points in line $\mathrm{L}$ will converge at one point, whose coordinate corresponds to parameters of L. In $(\rho, \theta)$ parameters space, a peak will form in the place of intersection through integral operation. By applying Hough transform, line detection in $(x, y)$ is converted into peak detection in parameters space.

\subsection{Wigner-Hough transform}

WVD is a bilinear time-frequency distribution, with extensive application. WVD of signal $S(t)$ is defined as

$$
W V D(t, \omega)=\int s\left(t+\frac{\tau}{2}\right) s^{*}\left(t-\frac{\tau}{2}\right) e^{-j \omega \tau} d \tau
$$

WVD has a good time-frequency gathered degree when it is applied to single component signal, which is from linear frequency modulation. While WVD is a bilinear method, when it is used to analyze multi-component signal, the cross terms brought by WVD will affect the detection of real signal In order to solve the problem of cross terms occurring in Wigner distribution of multi-component signal, Wigner-Hough transform is improved by combining the technology of signal processing and the method of image processing. The improved method will be used for signal detection to suppress the interference of cross terms. In the time-frequency plane, the WVD of signal $S(t)$ concentrates in a straight line. The equation is defined as: $v=f+g t$. So, the Wigner-Hough transform of analytical signal $S(t)$ was [7-10].

$$
T_{W H T_{S}}[s(t ; f, g)]=\int_{\infty}^{+\infty} \int_{\infty}^{+\infty} s\left(t+\frac{\tau}{2}\right) s^{*}\left(t-\frac{\tau}{2}\right) e^{-j 2 \pi(f+g t) \tau} d t d \tau
$$

Translated to polar form as shown in Fig. 1, expression for

$$
\begin{aligned}
T_{W H T_{S}}[s(t ; f, g)] & =\int_{-\infty}^{+\infty} \int_{-\infty}^{+\infty} s\left(t+\frac{\tau}{2}\right) s^{*}\left(t-\frac{\tau}{2}\right) e^{-j 2 \pi \frac{1}{\sin \theta}(\rho+t \cos \theta) \tau} d t d \tau \\
& =\int_{-\infty}^{+\infty} T_{W V D_{s}}\left(t, \frac{1}{\sin \theta}(\rho+t \cos \theta)\right) d t
\end{aligned}
$$

Equation (4) indicates that if $S(t)$ is a linear frequency modulation signal taken $f$ and $g$ as parameters, then integral value of $S(t)$ reaches the maximum. $f=\rho / \sin \theta, g=\tan \theta$. When parameters deviate from $f$ and $g$, integral value will diminish rapidly. So for certain linear frequency modulation signal, peak will appear in the corresponding parameters $(f, g)$ after applying WHT. And cross terms will be weakened through integral [9,11,12]. 
Table 1

Run-time comparison of three methods

\begin{tabular}{|c|c|c|c|c|c|c|}
\hline \multirow{2}{*}{$\begin{array}{c}\text { Sampling } \\
\text { points }\end{array}$} & \multicolumn{2}{|c|}{ WHT } & \multicolumn{2}{|c|}{ PWV-Hough } & \multicolumn{2}{|c|}{ Improved WHT } \\
\hline & $S N R / \mathrm{dB}$ & $\begin{array}{c}\text { Running } \\
\text { time/s }\end{array}$ & $S N R / \mathrm{dB}$ & $\begin{array}{c}\text { Running } \\
\text { time/s }\end{array}$ & $S N R / \mathrm{dB}$ & $\begin{array}{c}\text { Running } \\
\text { time/s }\end{array}$ \\
\hline \multirow{5}{*}{216} & -20 & 2.564 & -20 & 2.137 & -20 & 0.048 \\
\hline & -10 & 2.514 & -10 & 1.802 & -10 & 0.031 \\
\hline & 0 & 1.883 & 0 & 1.173 & 0 & 0.023 \\
\hline & 10 & 0.791 & 10 & 0.431 & 10 & 0.017 \\
\hline & 20 & 0.381 & 20 & 0.221 & 20 & 0.013 \\
\hline
\end{tabular}

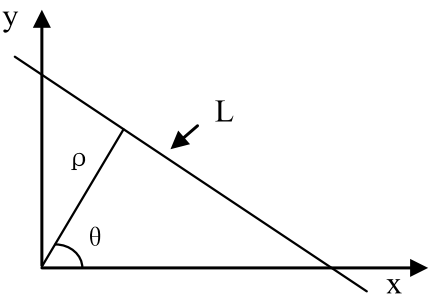

Fig. 1. Hough transform schematic.

\subsection{Sobel operator}

Sobel operator was a form of filtering operator which can achieve high effectivity using fast convolution function to extract image edge. For digital image $\{f(i, j)\}$, Sobel operators were defined as [6,13]:

$$
\begin{aligned}
A(i, j)= & \mid f(i-1, j-1)+2 f(i-1, j) \\
& +f(i-1, j+1)]-[f(i+1, j-1) \\
& +2 f(i+1, j)+f(i+1, j+1)] \mid \\
B(i, j)= & \mid f(i-1, j-1)+2 f(i, j-1) \\
& +f(i+1, j-1)]-[f(i-1, j+1) \\
& +2 f(i, j+1)+f(i+1, j+1)] \mid
\end{aligned}
$$

Where, $S(i, j)=\max (A(i, j), B(i, j)$ or $S(i, j)=A(i, j)+B(i, j)$. $A$ is used to detect horizontal edges and $B$ is used to detect vertical edges. Elements of the expression above are corresponding weighted factor. Selecting proper threshold $\eta$ and the following judgment will be: if $S(i, j)>\eta$, then $(i, j)$ was the edge point, $\{S(i, j)\}$ was the edged image. This edged image usually cannot be used directly because of data overflow, but the image is usually determined by its edge point and background. So it was binary image. Image edge obtained by using Sobel operator directly was rough and needed refining. In order to get refined edge detection, improved Sobel operator [14] was proposed in this paper. The maximum inhibition used in Canny edge detection algorithm and connection operation theory used in morphological were introduced into Sobel operator. The algorithm and specific steps can be seen in literature [6].

\subsection{Methods and steps}

So far we have given completed steps for extracting feature of gear fault signal based on Sobel operator and Wigner-Hough transform. They are as follows:

(1) Inputting vibrated signal;

(2) Calculating to obtain a Wigner-Ville distribution of signal;

(3) Taking Wigner-Ville distribution as image and using Sobel operator to complete edge detection;

(4) Extracting feature of fault signal by Hough transform.

\section{Simulation}

To prove the validity of this method, simulated signal of gear fault was firstly analyzed. According to the characteristics of gear fault signal, simulation signal was set as [15]:

$$
x(t)=\sum_{k=1}^{H} X_{k}\left[1+d_{k}(t)\right] \cos \left[2 \pi k z f_{z} t+\phi_{k}+b_{k}(t)\right]+i(t) ; \quad k=1,2, \ldots, H
$$



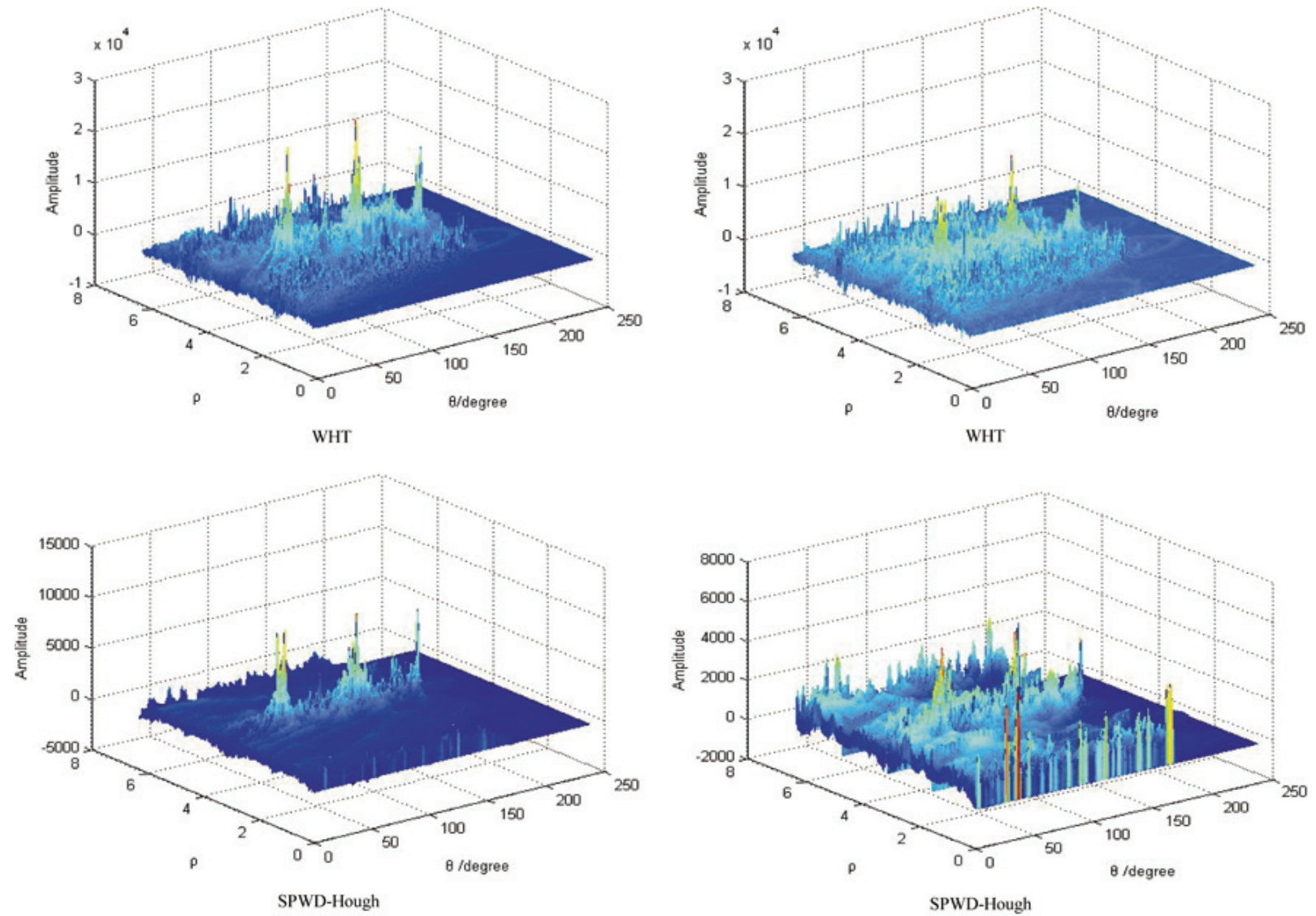

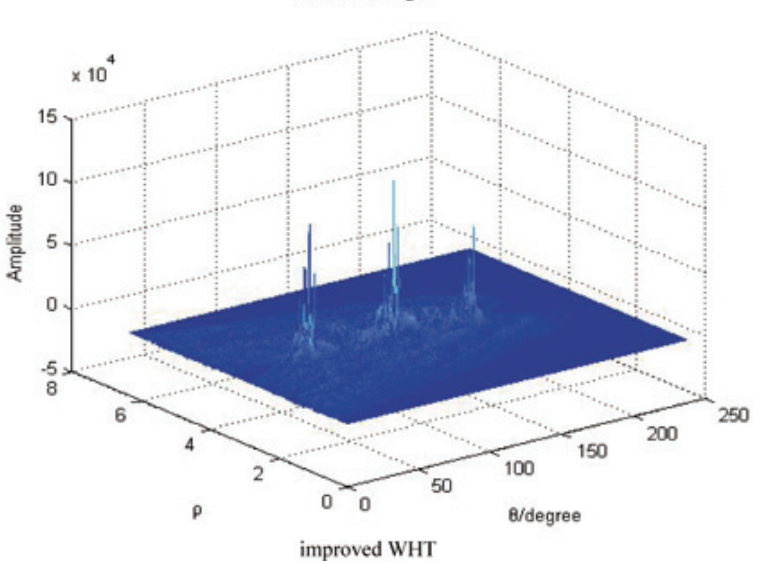

(a)

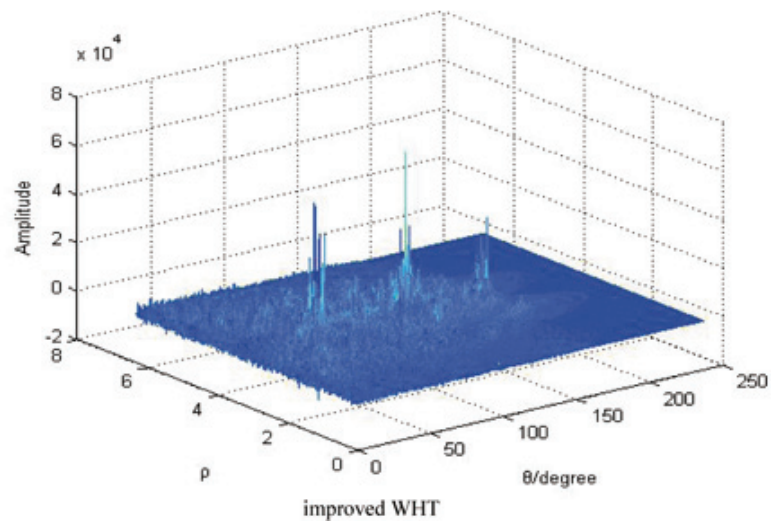

(b)

Fig. 2. Feature extraction of simulated signal with different $S N R$ using 3 kinds of method (a) $S N R=10 \mathrm{~dB}$ (b) $S N R=0 \mathrm{~dB}$ (c) $S N R=-10 \mathrm{~dB}$.

$X_{k}$ was amplitude of the $k$ order harmonic component of meshing frequency. $H$ was the number of polynomial terms in Eq. (7). $\varphi_{k}$ was the initial phase of $k$ order harmonic component of meshing frequency, $f_{z}$ was pivot frequency, $z$ was the number of teeth gears. $d_{k}(t)$ and $b_{k}(t)$ were modulation function of amplitude and phase. When gear have partial fault, the meshing was put into gear with axis every turn. So $d_{k}(t)$ and $b_{k}(t)$ were periodic function with rotational frequency of $f_{z}$ and its harmonics as repeated frequency, $i(t)$ was noise function.In simulation signal Eq. (7) as follows: $H=2, X=1.0, X_{2}=0.4, d_{1}(t)=0.2 \sin \left(2 \pi f_{z} t\right), d_{2}(t)=0.2 \sin \left(4 \pi f_{z} t\right), b_{1}(t)=$ $0.2 \sin \left(2 \pi f_{z} t\right), b_{2}(t)=0.2 \sin \left(4 \pi f_{z} t\right), \varphi_{1}=\varphi_{2}=\pi / 6, z=18, f_{z}=20 \mathrm{~Hz}$. The sampling frequency was 

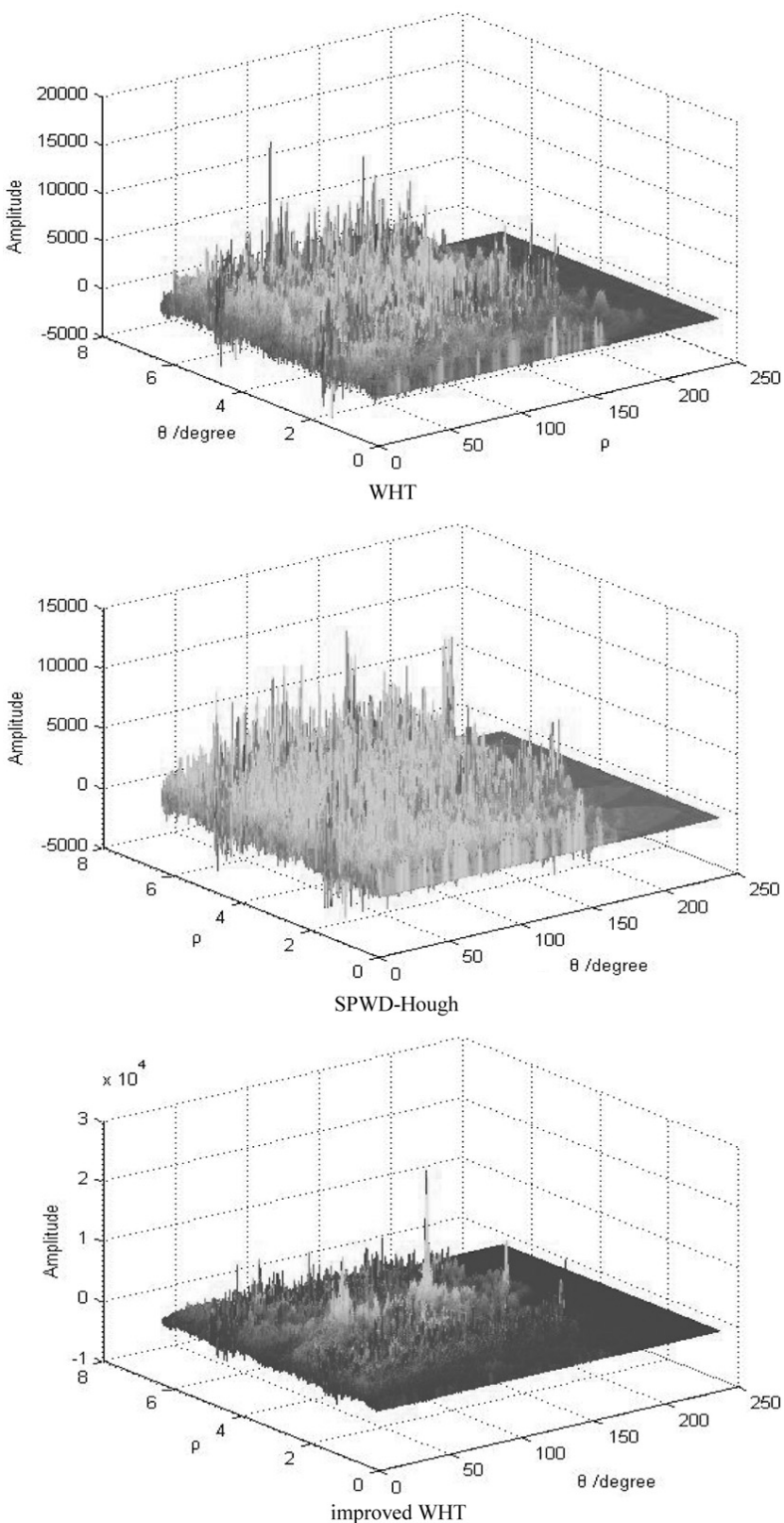

(c)

Fig. 2. (continued)

$4096 \mathrm{~Hz}$ and sampling points were 1000. As given in Fig. 2, simulation signal was compared respectively, with $S N R 0 \mathrm{~dB}, 10 \mathrm{~dB}$ and $-10 \mathrm{~dB}$. WHT, SPWD-Hough transform and improved WHT transform were applied to gain feature extraction of simulated signal.

It can be seen from Figs 2(a) and (b) that under condition of high $S N R(S N R=10 \mathrm{~dB}, 0 \mathrm{~dB})$, three methods can get correct characteristics of two FM signals, however, they also exhibit some obvious differences: (1) Due to the cross terms, burr was more by directly using WHT to extract feature; (2) SPWD-Hough transform is better to extract burr, but agglomeration was lowered somewhat; (3) Extraction map, got from improved WHT transform, made feature of two simulated signals shown clearly. The accuracy and aggregation were better than that of other two methods. By applying Hough transform, line detection of time-frequency distribution is converted into peak detection in 


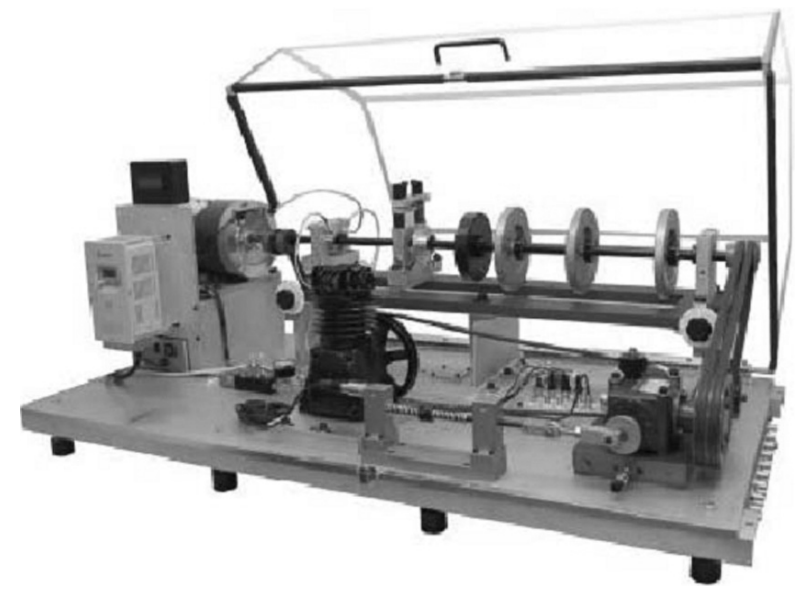

Fig. 3. Comprehensive fault simulation test bench.

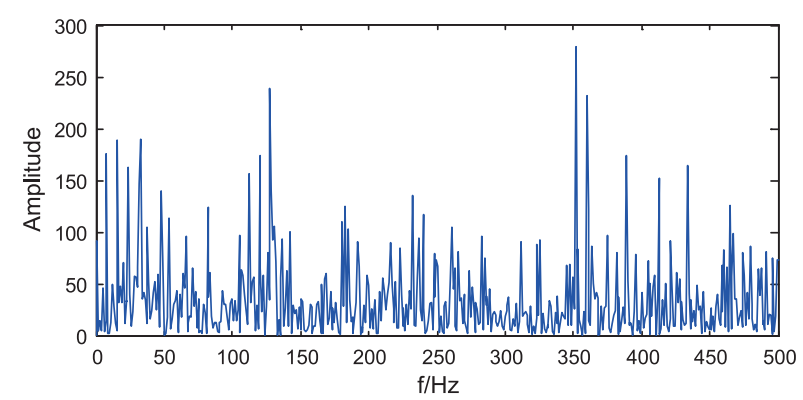

(a)

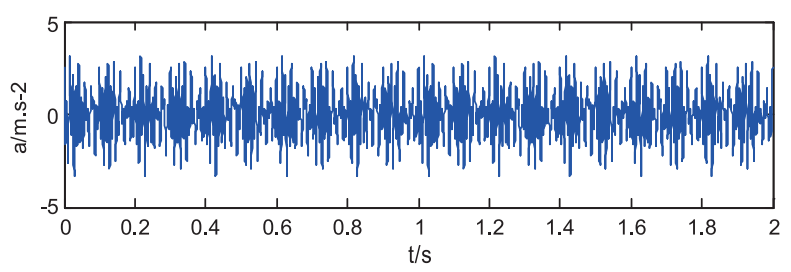

Fig. 4. Gears fault signal.

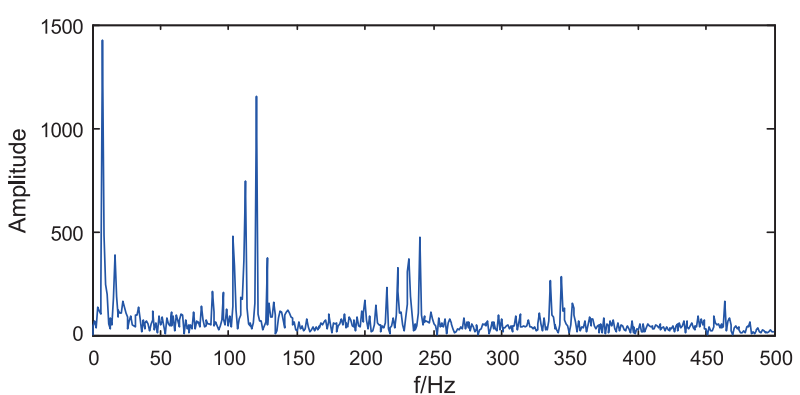

(b)

Fig. 5. Frequency signature of gears fault signal (a) Fourier spectrum (b) Envelope of the signal after processed by proposed method.

parameters space $(\rho, \theta)$.From the bottom picture of Figs 2(a) and (b), peaks of kinds of frequency component (including their harmonics or modulation frequencies) can be extracted clearly and its accuracy is high. According to Eq. (1), the corresponding frequency of each peak can be calculated (including $20 \mathrm{~Hz}$ and its harmonics, $360 \mathrm{~Hz}$, $720 \mathrm{~Hz}$ and their modulation frequencies).

Figure 2(c) showed the results in low $S N R(S N R=-10 \mathrm{~dB})$. It was shown in the top picture of Fig. 2(c) that the effect of cross terms and noise made it unlikely to get basic characteristics of signal by directly using WHT and there are many false peaks. Results were shown in the middle picture of Fig. 2(c), using Hough transforms to extract features after applying SPWD to signal. Although cross terms were largely inhibited by SPWD, this method still is unable to identify signal characteristics because noise was relatively large. By using method proposed in this paper, we firstly applied WVD and then used improved Sobel operator to detect edge, lastly used Hough transform to extract straight line. The results were shown in the bottom picture of Fig. 2(c). Compared the bottom picture of Fig. 2(c) with the bottom picture of Fig. 2(a), it can be seen that the effect of the former is not as good as that of the latter. But even in weak $S N R$, the two impacts can obviously be seen. Thus this method can effectively restrain interference of cross terms in WVD and was also reliable even in low SNR.

In this paper, comparisons were made towards different SNR in Table 1. It can be seen that under circumstances of same data length and same SNR, computing time of WHT was shortened greatly by using improved Sobel operator to test edge and efficiency is improved by nearly 2 orders of magnitude. For example, SNR dropped from $20 \mathrm{~dB}$ to $-20 \mathrm{~dB}$, direct running time of WHT increased from $0.381 \mathrm{~s}$ to $2.564 \mathrm{~s}$. But running time of WHT after filtering by applying improved Sobel operator was increased from $0.013 \mathrm{~s}$ to $0.048 \mathrm{~s}$. The calculation speed was greatly improved and speed change range was very small by improved method. It was clear that the improved WHT method was very suitable for real-time feature extraction of fault signal even in large amount of data and low SNR. 


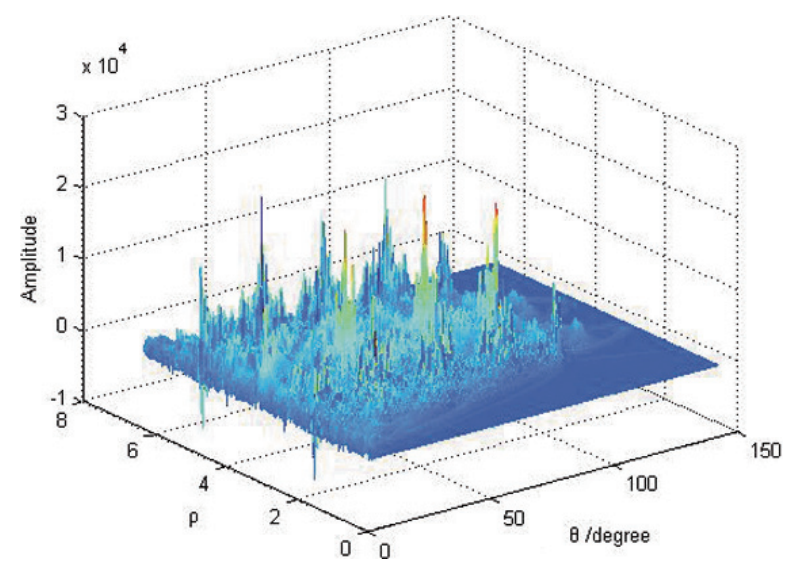

(a)

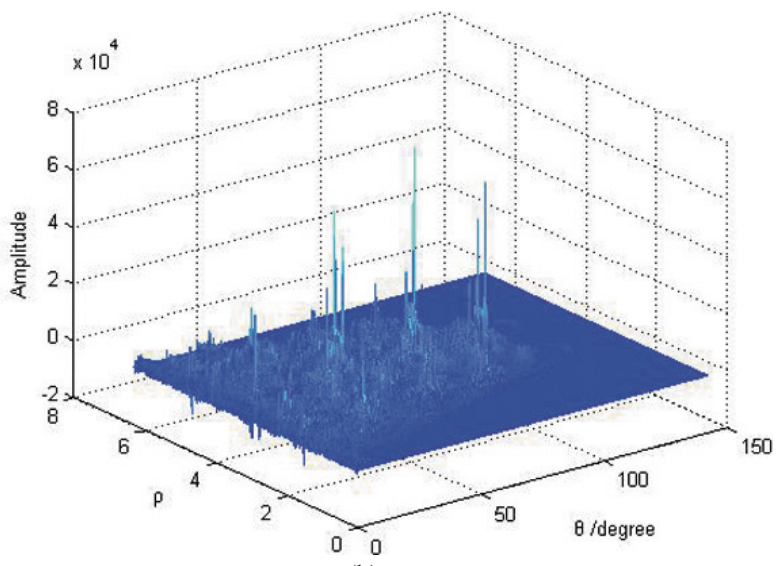

(b)

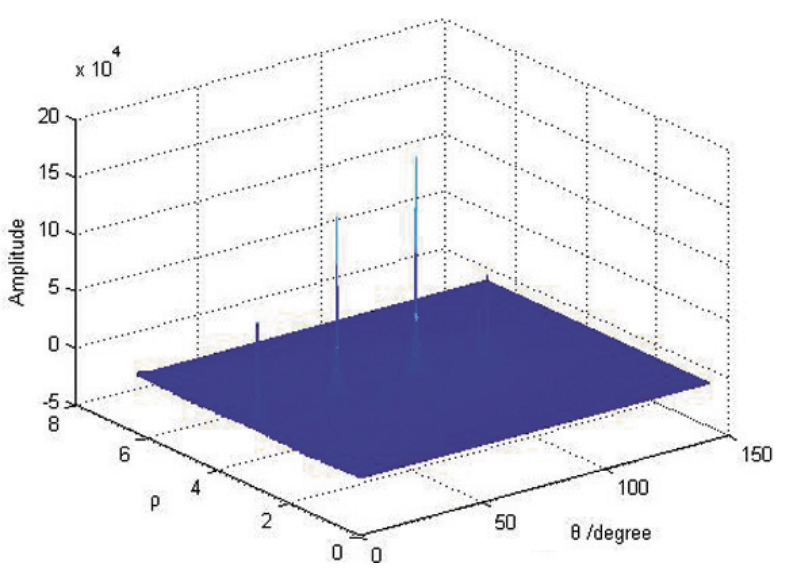

(c)

Fig. 6. Feature extraction of gears fault signal (a) Based on WHT (b) Based on the improved WHT (c) Feature extraction before adding a small crack.

\section{Experiment research}

To further prove the effectiveness of feature extraction, fault gear with root crack was analyzed. Gear fault diagnosis test was implemented by using the comprehensive fault simulation test bench (MFS-MG). The test bench is composed of motor, a mechanical transmission device, sensor, the hardware circuit, computer and related software. Test bench was shown in Fig. 3. The number of teeth of driving wheel is $z_{1}=10$, motor speed is $f_{r 1}=720 \mathrm{r} / \mathrm{min}$. The number of teeth of driven wheel gear is $z_{2}=15$, module is $m=2 \mathrm{~mm}$, rotating frequency of gear axis is $f_{r 2}=480 \mathrm{r} / \mathrm{min}$, meshing frequency is $f_{z}=120 \mathrm{~Hz}$ and sampling frequency is $1 \mathrm{kHz}$. A gear with defects has been considered and we have added a small crack to one gear. In the gear, a small groove, with $0.1 \mathrm{~mm}$ width and $3 \mathrm{~mm}$ deep, was processed to simulate tooth crack. The time-domain waveform of gearbox vibration signal was shown in Fig. 4, from which it can be found that cyclical pulse signal was almost submerged in the strong background noise. Fourier spectrum of fault signal was shown in the Fig. 5(a). Obviously, interference brought great difficulties to extract fault signal feature.

Figure 6 showed feature extraction using the WHT and improved WHT and described the effect to add a crack to worn gears. In Fig. 6(a), because of the influence of noise and cross terms, burr was much and its amplitude is larger when directly using WHT to extract feature which affected fault diagnosis seriously. Figure 6(b) was feature extraction figure using improved WHT transform. The results, from before adding a small crack to one gear, have been presented in Fig. 6(c) for comparison. In the polar coordinate $(\rho, \theta)$ parameters space, the peak corresponding frequency(including $8 \mathrm{~Hz}$, the wheel frequency, the harmonics of the gear mesh frequency $(240,360 \mathrm{~Hz})$ and their 
modulation frequencies around them)can be seen in the Fig. 6(b). Figure 5(b) showed the envelope of the signal after processed by proposed method. Obviously, Figs 5(b) and 6(c) clearly indicated the characteristics of fault signal and it provided further conditions for fault diagnosis. It confirmed that this method can effectively extract gear fault feature in low noise ratio.

\section{Conclusions}

According to the facts that the different components of signal have different distribution characteristics in the time-frequency plane, we can achieve the goal of parameter extraction using Hough transform. In the time-frequency plane, real signal energy is concentrated in the corresponding frequency line and is always positive. After applying Hough transformation, a sharp peak will be formed in the parameter space. While the amplitude of cross terms alternates positive or negative. After applying Hough transformation, the forming peak is very small. Noise and interference has a random and dispersive distribution. The forming peak point is small and dispersed after applying Hough transformation. For fault signal, its frequency component varies with time rule and its energy distribution is concentrated in a straight line. Through Hough transformation, a larger peak can be formed. So, the fault signal can be identified in the parameter space according to the difference of peak.

This method, which regards fault signal time frequency spectrum as $2 \mathrm{D}$ image and uses image processing method to conduct analysis and state recognition, was effectively verified by many literatures. Sobel operator was firstly introduced to detect edge for time frequency distribution and Hough transform to extract signal features. The simulation and experimental results demonstrated the effectiveness of this method. Improved WHT algorithm can restrain cross terms produced by WVD in analyzing multi-component signal, especially in low SNR cases. Improved WHT can better inhibit the influence of noise and the algorithm is fast calculation speed and is very suitable for feature extraction of large quantities data and low SNR fault signal.

\section{Acknowledgments}

The authors express their appreciation for the ?nancial support provided by the State 863 Project of China (Project No: 2006AA06Z10), natural science foundation of Hunan Province (Project No: 12JJ4034) and Fund of the 11 $1^{\text {th }}$ Five-year Plan for Key Construction Academic Subject (Optics) of Hunan Province.

\section{References}

[1] S. Serhat and A. Emine, Feature extraction related to bearing damage in electric motors by wavelet analysis, Journal of the Franklin Institute 340 (2003), 125-134.

[2] G. Chen and J. Cao, Statistical performance of the Wigner distribution and cross Wigner-Ville distribution, Journal of Shanghai University: English Edition 7(4) (2003), 379-383.

[3] H. Li, Y. Zhang and H. Zheng, Hilbert-Huang transform and marginal spectrum for detection and diagnosis of localized defects in roller bearings, Journal of Mechanical Science and Technology 23(2) (2009), 291-301.

[4] J. Lin and L. Qu, Feature extraction based on Morlet wavelet and its application for mechanical fault diagnosis, Journal of Sound and Vibration 234(1) (2000), 135-148.

[5] D. Yu, J. Cheng and Y. Yang, Application of Hilbert-Huang transform method to gear fault diagnosis, Chinese Journal of Mechanical Engineering 41(6) (2005), 102-107.

[6] L. Shen, X. Zhou, L. Liu et al., Application of morphological wavelet de-noising in extracting gear fault feature, Transactions of the Chinese Society for Agricultural Machinery 41(4) (2010), 217-221.

[7] H. Li, Z. Zhang and Z. Guo, Rolling bearing fault diagnosis using hough transform of time-frequency image, Journal of Vibration, Measurement \& Diagnosis 30(6) (2010), 634-639.

[8] F. Liu, D. Sun, Y. Huang et al., Multi-component LFM signal feature extraction based on improved wigner-hough transform, Transactions of Beijing Institute of Technology 28(10) (2008), 914-917.

[9] R.B. Randall, Detection and diagnosis of incipient bearing failure in helicopter gear boxes, Engineering Failure Analysis 11 (2004), 177-190.

[10] H. Li, H. Zheng and L. Tang, Wigner-ville distribution based on EMD for faults diagnosis of bearing, Lecture Notes in Computer Science 4223 (2006), 803-812. 
[11] L. Stankovic and S. Stankovic, An analysis of instantaneous frequency representation using time-frequency distributions generalized Wigner distribution, IEEE Transon Signal Processing 43(2) (1995), 549-552.

[12] J. Liu, J. Yang and J. Zhou, Detection performance of linear frequency modulated signals based on Wigner-Hough transform, Signal Processing 21(4A) (2005), 546-549.

[13] S. Barbarossa and A. Zanalda, A combined Wigner-Ville and Hough transform for cross terms suppression and optimal detection and parameter estimation, IEEE International Conference on Acoustics, Speech and Signal Processing 5 (1992), 173-176.

[14] M. Niu, G. Liu and D. Liu, Sobel operator based cross-terms attenuation in the Wigner-Ville distribution, Journal of Astronautic Metrology and Measurement 27(2) (2007), 38-40.

[15] B. Li, P. Zhang, G. Ren et al., Gear fault diagnosis using empirical mode decomposition, genetic algorithm and support vector machine, Journal of Vibration, Measurement \& Diagnosis 29(4) (2009), 445-449. 

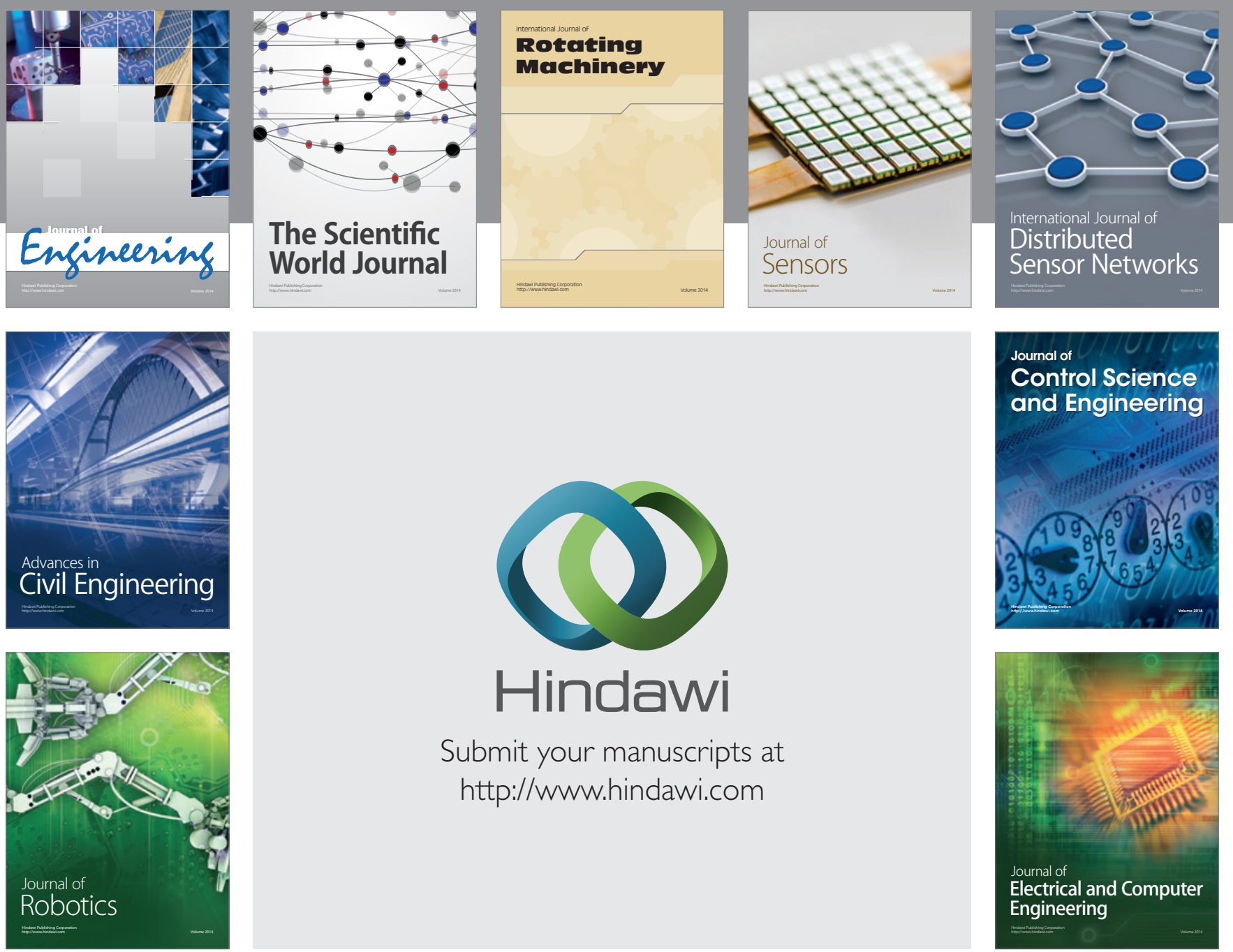

Submit your manuscripts at

http://www.hindawi.com
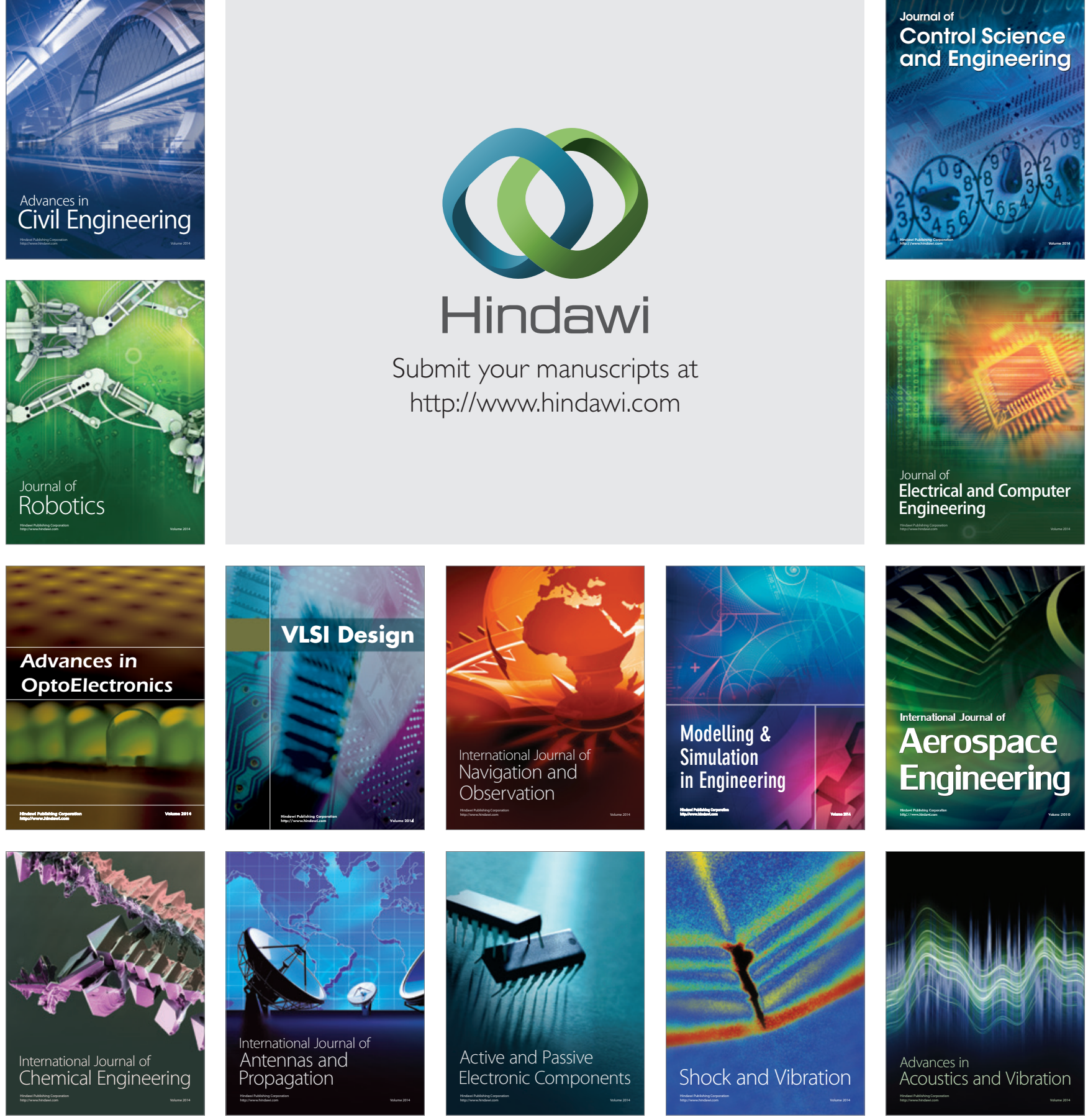